\title{
Onychomycosis Treatment with FotonaLaser
}

\author{
Aristides Arellano Huacuja, Dafne ArellanoMontalvo, Anja Arellano Montalvo
}

Dermatological Clinic and Aesthetic Surgery of Puebla. Puebla, Mexico

Received: July 05, 2021; Accepted: July 15, 2021; Published: July 21, 2021

*Corresponding author: Arístides Arellano Huacuja,PlasticSurgery, Clinic of Dermatology and AestheticSurgery of Puebla, Puebla, Pué, Tel. No: (222)437740, Fax: (222)2377494, E-mail: aristidesarella@yahoo.com.mx

\section{Introduction}

Onychomycosis is a fungal infection of the nails. It does not usually cause discomfort, but it alters the aesthetics of the affected nails and, if not treated in time, it can become chronic and spread. The cause of onychomycosis are fungi of the dermatophyte type. Onychomycosis is the disease that most frequently affects the nails of humans (50\%), being responsible for more than half of the cases of nail alteration. Studies show prevalence figures between $2 \%-18 \%$ of the population. It manifests in the form of onychodystrophy or alteration of the normal appearance of one or more fingernails or toenails. Toenails are affected more often than fingernails. It is very common to affect several nails at the same time. Clinically there may be onycholysis, subungual hyperkeratosis with desquamation, dyschromia ranging from yellowish or greenish to brown or black. The treatment of onychomycosis may require taking oral antifungal drugs (undecenoic acid, azoles and sometimes allylamines) combined with long-term treatment ( 2 to 18 months), they can cause liver damage, kidney failure, anemia, thrombocytopenia, neutropenia, gastrointestinal disorders and alopecia among other diseases. In addition, other treatments can be performed, ranging from surgery (laminectomy, matrixectomy), onychoabrasion, to the use of new therapies such as: photodynamic therapy, iontophoresis, and lasers today. The laser is a revolutionary treatment for Onychomycosis. It is a simple and effective procedure that uses Neodymium or Erbium: Yag laser to act to the depths of the damaged nail and the underlying skin, weakening and eliminating the fungus that has invaded the patient's nail.

The body's natural defense process is stimulated to regenerate the nail in a normal way. Compared with traditional methods, the laser does not damage the tissue and is not limited to its chemical action like topical drugs, nor does it risk causing liver damage like oral medications. By applying the Fotona Laser with smoothmode technology throughout the nail plate, we achieve, through heat irradiation, eradicate any fungus or germ lodged in the nail. Without side effects, fast, simple and $100 \%$ safe.

\section{Fotona laser treatment}

The laser is done in 3 simple steps:

Step 1: Thin the nail:

In the presence of infection, the nail becomes deformed and thickened. Thinning the nail allows the laser to act down to the deepest layers and thus make the treatment more effective.

Step 2: Administer the pulses of the Nd: YAG or Erbium: Yag laser in a circular shape that helps cover the entire nail including the nail bed area. The patient will present a sensation of heat in the nail and sometimes pain.

Step 3: Place the Nd: YAG / Erbium: Yag laser in the peripheral area of the nail and around the finger and / or foot. This in order to completely eliminate the fungus from the areas near the affected nail.

The Erbium: Yag laser is as effective as the Neodymium: Yag laser in the elimination of the fungus, with both light sources the elimination is constant.

The Fotona laser has disinfection properties offering superior clinical results, thermally and selectively affects the deeper skin and tissue structures.

The natural growth of the nail and the immune system itself will restore the nail to its natural and healthy appearance.

Advantage

- There are no side effects

- Without pain

- Fast and safe

- Immediate return to daily activities

- Better results compared to traditional treatments

\section{Parameters}

A long-pulse Nd: YAG laser with a wavelength of $1064 \mathrm{~nm}$ is used, with fluences ranging from 35 to $40 \mathrm{~J} /$ square $\mathrm{cm}$. A spot size of $4 \mathrm{~mm}$ in diameter, a pulse duration of $35 \mathrm{~ms}$, and a frequency of $1 \mathrm{~Hz}$ [1-3]. Applying the entire nail plate in a spiral manner, a two-minute pause is performed, and the treatment is repeated. The pause is repeated two more times for a total of three passes. Each session lasts approximately 15 minutes. One session a week is established during a 5 -week cycle to achieve the best possible results. However, it will be the doctor who determines exactly the number of sessions appropriate for each case.This procedure does not require any type of anesthesia, antibiotic, or posttreatment analgesic.In places where the heat and humidity of the climate are a constant, it is frequent that these types of diseases 


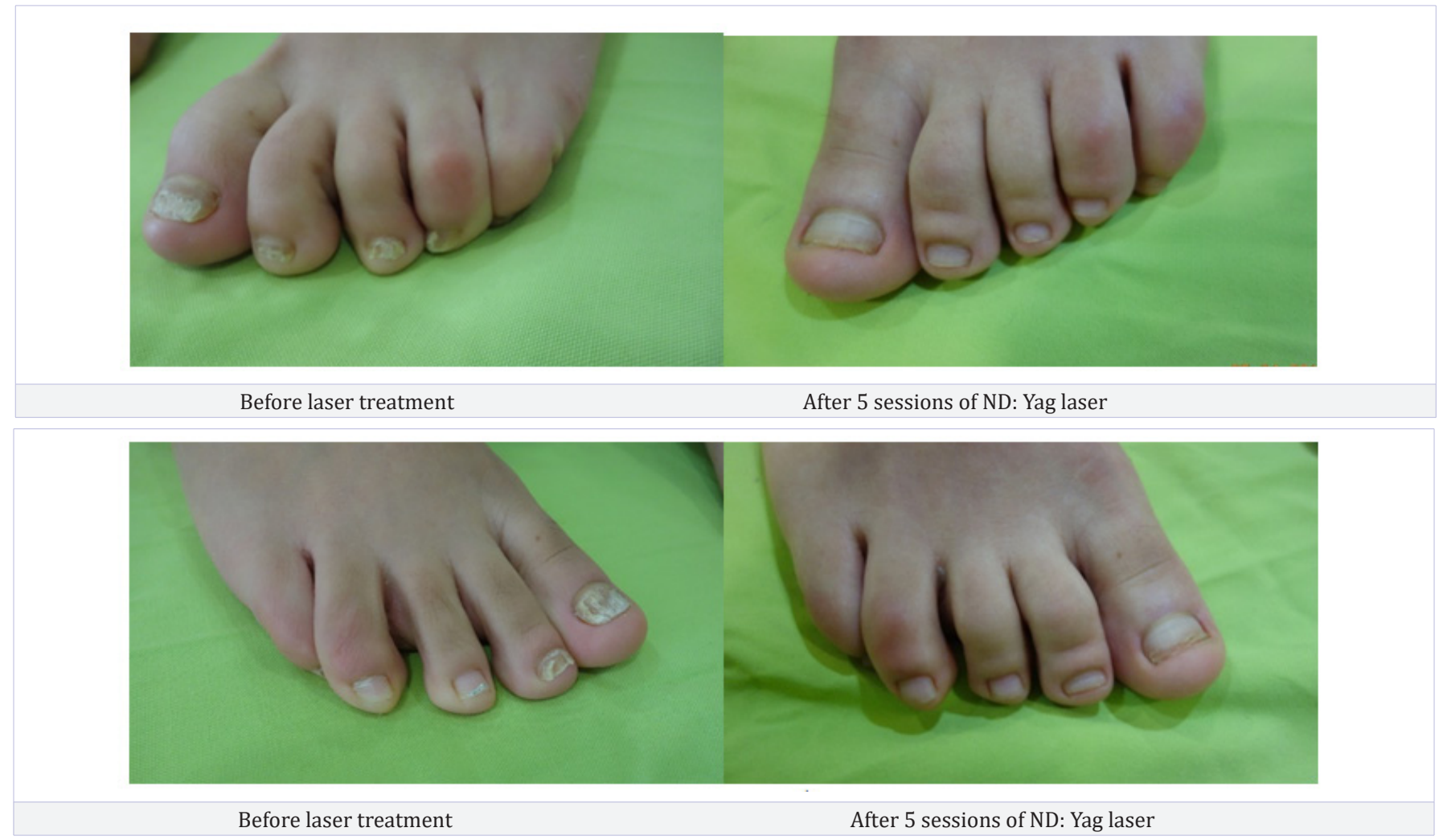

appear. Other factors such as aging. Keep the foot moist, suffer from ringworm in the foot, psoriasis, diabetes, relatives with onychomycosis or Immunosuppression.Finally, with the intention of preventing reinfection in susceptible patients, a change in hygiene and footwear habits is recommended, in addition to the application of deodorants and fungicides for local use in powder

\section{References}

1. IT of the National Health System. Onychomycosis: diagnosis and treatment Julián Larruskain Garmendia. Head of Section. Microbiology Service. Donostia Hospital. 2008;32(3).

2. V Preciado Román, E Úraga Pazmiño. Onychomycosis treated with laser, clinical trial. Private Skin Center Dr. Enrique Uraga Peña.

3. Fasano, Kiernan, Verea, Pecotche, Fasano, Patricia. Onychomycosis Retrospective study on epidemiology and treatment. Hospital San Juan de Dios. 2009. 